Proceedings of the 48th International School and Conference on the Physics of Semiconductors "Jaszowiec 2019"

\title{
The Influence of Oxygen and Carbon Contaminants on the Valence Band of $\boldsymbol{p}-\mathrm{GaN}(0001)$
}

\author{
D. Majchrzak ${ }^{a, b, *}$, M. Grodzicki ${ }^{a, c}$, P. Ciechanowicz ${ }^{a, c}$, J.G. Rousset $^{a}$, \\ E. PiskorsKA-HOMMEL ${ }^{a, b}$ AND D. HOMMEL ${ }^{a, c}$ \\ ${ }^{a}$ Łukasiewicz Research Network - PORT Polish Center for Technology Development, \\ Stabłowicka 147, Wrocław, Poland \\ ${ }^{b}$ Institute of Low Temperature and Structure Research, Polish Academy of Sciences, \\ Okólna 2, 50-422 Wrocław, Poland \\ ${ }^{c}$ Institute of Experimental Physics, University of Wrocław, Pl. M. Borna 9, Wrocław, Poland
}

\begin{abstract}
Mg-doped GaN(0001) epitaxial layers, grown by molecular beam epitaxy in a setup interconnected with an analytic chamber, were analysed by means of X-ray photoelectron spectroscopy to study their physicochemical properties under different preparation methods. Investigations were carried out for the following samples: asgrown, air-exposed and treated with isopropanol (IPA) or $\mathrm{HCl}$, and in situ cleaned. The X-ray photoelectron spectroscopy results confirmed that the air-exposed samples are contaminated with oxygen and carbon atoms, which can be removed only by in situ cleaning. The valence band maximum varies in the range from $1.2 \mathrm{eV}$ to $2.7 \mathrm{eV}$ below the Fermi level for the as-grown and ex situ prepared samples, respectively. The valence band maximum for in situ cleaned sample is located at $1.7 \mathrm{eV}$ below the Fermi level.
\end{abstract}

DOI: $10.12693 /$ APhysPolA.136.585

PACS/topics: $68.47 . \mathrm{Fg}, 73.20 . \mathrm{At}$, 82.80.Pv

\section{Introduction}

GaN-based materials attract great interests in the electronic industry because of their wide and direct energy band gap $(3.4 \mathrm{eV})$ and a good thermal conductivity $[1,2]$. These semiconductors have a potential to fabricate the highest standard electronic devices working in various areas, such as light emitters and high-power devices [3-6]. The condition of the output materials in the production process has an impact on the final performance of such devices because electronic properties of GaN(0001) depend on the surface quality. Thus, a native oxide layer formed on the surface after air exposure impacts properties of the metal/GaN interface [7]. Several methods are applied to prepare the surface of the output substrate: etching in acids (e.g. $\mathrm{HCl}, \mathrm{HF}$ ) or alkalis (e.g. $\mathrm{KOH})[8]$, annealing in vacuum, and ion bombardment $[9,10]$. Comparative results show that $\mathrm{HCl}$ is the most efficient concerning surface oxygen coverage decrease and HF for carbon reduction [11]. GaN surfaces treated with $\mathrm{HCl}$ are contaminated with $\mathrm{Cl}$ atoms which occupy dangling bonds left by some of the oxygen atoms and prevent re-oxidation process [10,12]. Given that wet etching techniques cannot completely remove the oxygen and carbon contamination, methods such as annealing under ultrahigh vacuum (UHV) or an ion bombardment need to be introduced to obtain clean surfaces.

\footnotetext{
* corresponding author; e-mail: dominika.majchrzak@port.org.pl
}

The ion bombardment with $\mathrm{Ar}^{+}$or $\mathrm{Xe}^{+}$introduces defects and preferentially remove nitrogen atoms [13]. To prevent the nitrogen depletion low energy $\mathrm{N}^{+}$-ion bombardment may be implemented [14] or combined techniques including ion bombardment followed by a short annealing [11].

The influence of all mentioned preparation techniques on surface properties of $p$-GaN epitaxial layers have been studied before [8-10]. However, these studies omitted the explicit measurement of the valence band maximum (VBM) position. Moreover, no comparative analysis with the as-grown sample, being free of any contamination, has been done so far. In the literature contradictory informations appear such as different VBM and Ga $3 d$ peak shifts after $\mathrm{HCl}$ treatment $[15,16]$. Therefore, our aim is to take a closer look at the role of native contaminants on $p$-GaN surfaces, and in particular, to answer how different preparation methods impact the physicochemical properties, especially the Fermi level $\left(E_{\mathrm{F}}\right)$ position relative to the VBM of $p$-GaN.

\section{Experimental details}

Mg-doped GaN sample with $\mathrm{Mg}$ concentration $5 \times 10^{19}$ atoms $/ \mathrm{cm}^{3}$ (determined from secondary-ion mass spectrometry measurements) was grown in a molecular beam epitaxy chamber with a base pressure of $5 \times 10^{-12}$ mbar, on a commercial $5 \mu \mathrm{m}$ thick, nondoped MOCVD GaN template deposited on $\mathrm{Al}_{2} \mathrm{O}_{3}$. During growth reflection high-energy electron diffraction (RHEED) technique was used to monitor the crystalline quality of the sample. RHEED patterns obtained for as-grown $p$-type GaN revealed stripes corresponding to 
the $(1 \times 1)$ wurtzite structure. After growth the GaN sample was transferred under UHV into the analysis chamber and X-ray photoelectron spectroscopy (XPS) for the asgrown sample (without exposure to contamination) was performed. All measurements as well as the transfer of the as-grown sample were carried out at a pressure lower than $5 \times 10^{-11}$ mbar. Then the sample was exposed to air and cut into pieces of $1 \times 1 \mathrm{~cm}^{2}$ in size, which were subjected to various cleaning procedures: (1) isopropanol (IPA), (2) $\mathrm{HCl}$ treatments, and (3) in situ cleaning by $\mathrm{Ar}^{+}$-ion bombardment with and without annealing up to $800^{\circ} \mathrm{C}(2 \mathrm{~min})$. Before the placement into the analysis chamber, samples were rinsed with de-ionized water and blow-dried with $\mathrm{N}_{2}$. Then they were degassed in the UHV by annealing up to $500^{\circ} \mathrm{C}$ for $10 \mathrm{~min}$. Temperature was measured using a K-type thermocouple. Surface electronic and chemical conditions of the samples before and after surface treatments were analysed using XPS with a monochromatic $\mathrm{Al} K_{\alpha}(1486.7 \mathrm{eV})$ radiation source. Photoelectrons were collected with a hemispherical electron energy analyzer (Argus CU) with a step size of $0.05 \mathrm{eV}$ and a pass energy of $20 \mathrm{eV}$. Binding energy (BE) values were referenced to $E_{\mathrm{F}}$ whose position was calibrated using a cleaned Ag sample. Measurements were made at a take-off angle of $30^{\circ}$ with respect to the surface and at room temperature.

\section{Results and discussion}

The XPS measurements (Fig. 1) show that the asgrown $p$-GaN is free of oxygen and carbon contaminants unlike the air-exposed samples subjected only to chemical treatment. The spectrum for $p$-GaN after IPA procedure can be regarded as the spectrum for air-exposed sample, because the experiment has shown that IPA procedure does not shift VBM and does not significantly change the amount of oxygen and carbon contaminants compared to air exposed samples. The $\mathrm{O} 1 \mathrm{~s}$ peak located at $\mathrm{BE}$ of $531.9 \mathrm{eV}$ had the highest intensity for the IPA sample (Fig. 1a). Its position shifted to $532.1 \mathrm{eV}$ and the oxygen amount noticeably decreased for the $p$ $\mathrm{GaN}$ treated with $\mathrm{HCl}$. Trace amounts of oxygen completely disappear for samples cleaned by $\mathrm{Ar}^{+}$-ion bombardment in the UHV chamber. As shown in Fig. 1b, the similar amount of carbon was found in samples which underwent IPA or $\mathrm{HCl}$ procedures. The $\mathrm{C} 1 s$ line has a position of the binding energy equal to $285.2 \mathrm{eV}$. Carbon was not detected for the $\mathrm{Ar}^{+}$-ion bombarded sample.

The evolution of the valence band for $p$-GaN(0001) samples as a function of different surface treatments is shown in Fig. 2. The results indicate that the VBM of the as-grown sample is located $1.2 \mathrm{eV}$ below $E_{\mathrm{F}}$ and is shifted by about $1.5 \mathrm{eV}$ towards higher $\mathrm{BE}$ for the airexposed samples. The VBM lies at a BE of $2.7 \mathrm{eV}$ for the IPA sample and is slightly shifted for the $\mathrm{HCl}$ treated $p$ GaN layer. One can see that the $\mathrm{Ar}^{+}$-ions bombardment significantly changes the valence band. In the vicinity of

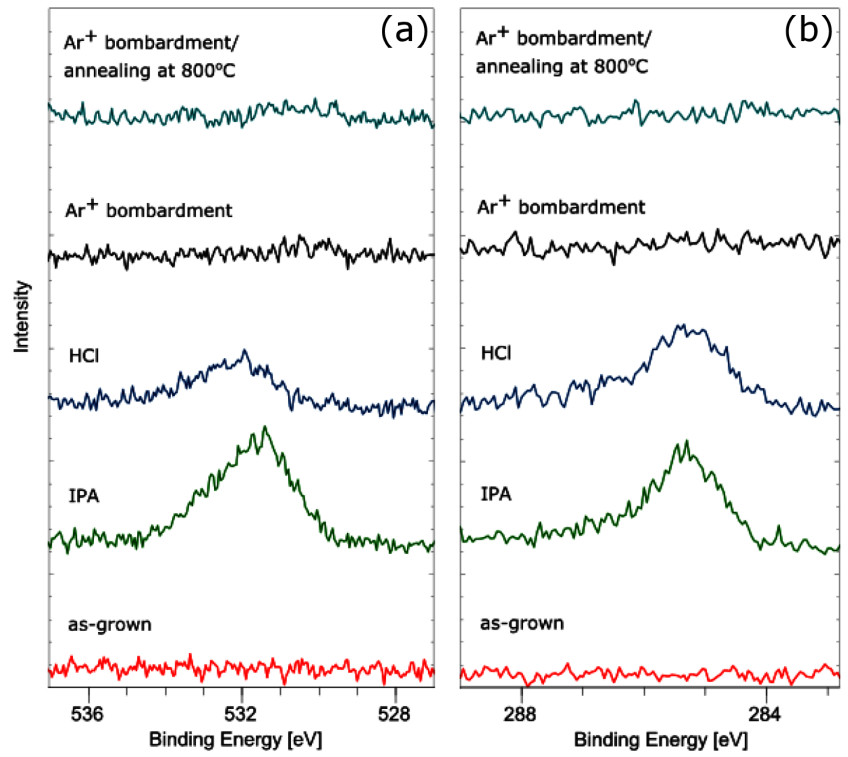

Fig. 1. The XPS spectra of (a) the O $1 s$ and (b) C $1 s$ core level lines for the $p$-GaN(0001) sample after different surface treatments.

the Fermi level the electron density of states increases. Thus, the energy position of the valence band edge is difficult to estimate. This is due to the fact that $\mathrm{Ar}^{+}$-ions preferentially sputter nitrogen atoms which leads to gallium enrichment of the surface [13]. Therefore annealing up to $800^{\circ} \mathrm{C}$ was applied to compensate the loss of $\mathrm{N}$ atoms by desorbing the excess of $\mathrm{Ga}$ and afterwards the VBM was located at $1.7 \mathrm{eV}$. The shift of VBM from about $2.7 \mathrm{eV}$ to the mentioned value results from a reduction of oxygen and carbon contaminants on the surface. It should be noted that the result obtained for the sample after the ion bombardment with annealing is the closest to the as-grown sample, however the VBM value after this surface treatment is still higher by $0.4 \mathrm{eV}$. In Fig. 3 positions of the Ga $3 d$ peaks are shown. One can see that the $\mathrm{Ga} 3 d$ line shifts similarly to the VBM. The Ga $3 d$ state for the as-grown $p$-type $\mathrm{GaN}$ has a $\mathrm{BE}$ at $18.95 \mathrm{eV}$ and after air-exposition its position is shifted to higher binding energies by ca. $1.5 \mathrm{eV}$. Next, for the $\mathrm{Ar}^{+}$-ions bombardment the $\mathrm{Ga} 3 d$ is located at a $\mathrm{BE}$ of $19.2 \mathrm{eV}$ and $19.45 \mathrm{eV}$ followed by annealing. The energy distance between the Ga $3 d$ peak and the VBM for all samples oscillates around $17.75 \mathrm{eV}$, which is consistent with previously reported results $[14,17,18]$.

The theoretical study by Segev and Van de Walle shows that at the $\mathrm{GaN}(0001)$ surface two singularity of surface density of states (SDOS) can appear [19, 20]. The upper state is located at $\approx 2.8 \mathrm{eV}$ and the lower at $\approx 1.7 \mathrm{eV}$ above the VBM. According to the calculation, in the case of non-doped GaN the higher states are empty, while the lower states are full and their occupation depends on the conductivity type and the doping concentration [19-21]. The Fermi level pinning at these states was experimentally observed by Janicki et al. in Ref. [22]. 


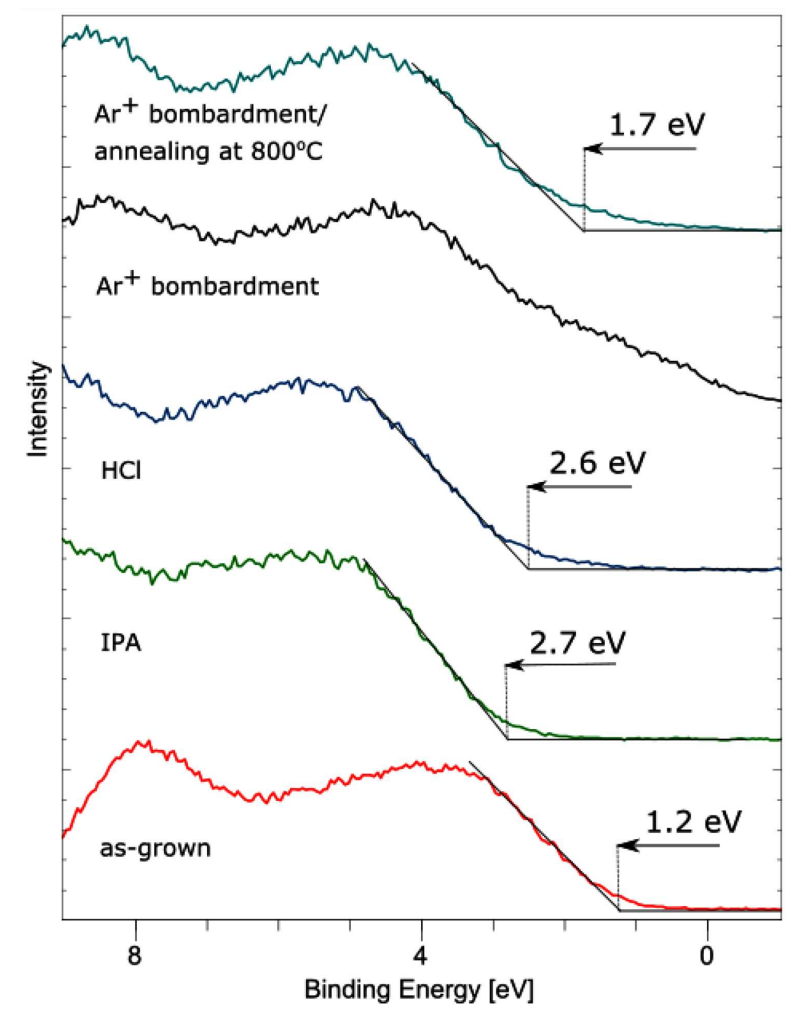

Fig. 2. The XPS spectra of the valence band for the $p$-GaN(0001) sample after different surface treatments.

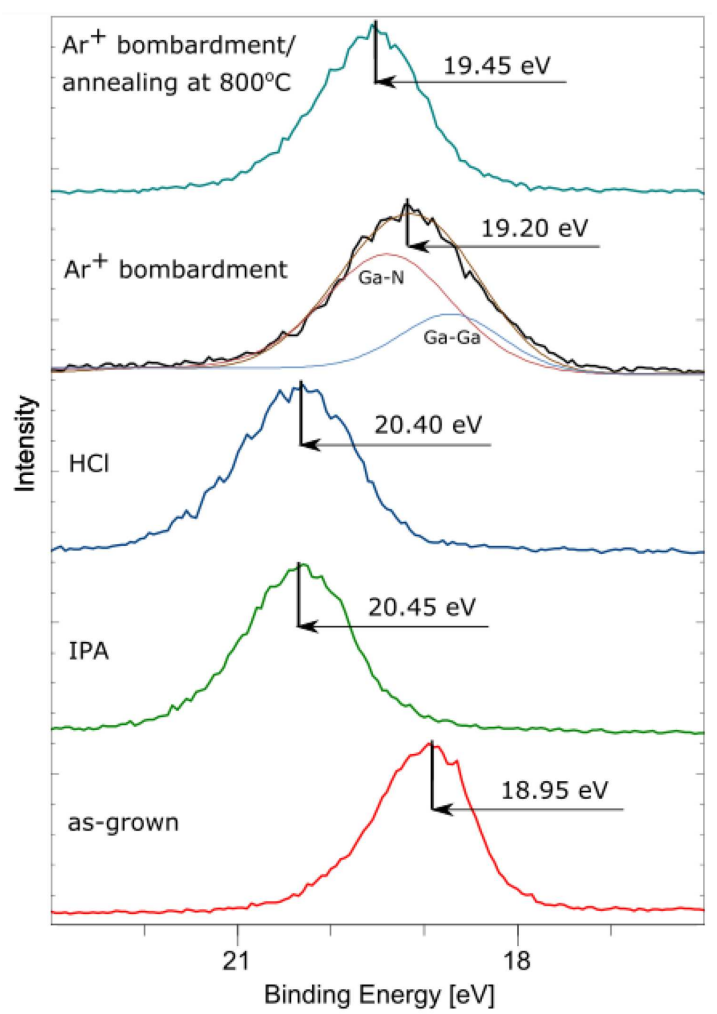

Fig. 3. The XPS spectra of the Ga $3 d$ state for the $p$-GaN(0001) sample after different surface treatments.
Based on our results we can state that the presence of oxygen atoms adsorbed at the $p$-type GaN surface impact the electronic properties of the surface which leads to the pinning of $E_{\mathrm{F}}$ at the upper, empty state which in consequence leads to emerge the inversion layer. Furthermore, the $1.2 \mathrm{eV}$ distance between the $\mathrm{VBM}$ and $E_{\mathrm{F}}$ for the as-grown sample is lower than expected from Segev and Van de Walle's calculation [19] and Janicki's measurements [22]. This can be a result of the fact that the lower state is not fully occupied and/or this difference may arise from the surface photovoltage effect appearing during XPS and UPS measurements, which was noted by Long and Bermudez [21]. The VBM for the air-exposed sample and $\mathrm{Ar}^{+}$-ions bombarded is located at $1.7 \mathrm{eV}$ below $E_{\mathrm{F}}$, which is in good agreement with the DFT calculation and Janicki's measurements [22]. This may also stem from the fact that on the ion bombarded surface the photovoltage effect can be weaker or even does not occur at all. This is also consistent with other works [14, 23], where $\mathrm{N}^{+}$-ion bombardment was applied.

\section{Conclusions}

The XPS technique was used to study physicochemical properties of the $p$-type $\mathrm{GaN}(0001)$ surface just after growth and air exposure followed by different surface preparations (isopropanol, $\mathrm{HCl}, \mathrm{Ar}^{+}$-ion bombardment). The valence band maximum (VBM) relative to the Fermi level position is $1.2 \mathrm{eV}$ for the as-grown sample and $c a$. $2.7 \mathrm{eV}$ for the samples air-exposed. The sample cleaned in situ by $\mathrm{Ar}^{+}$bombardment followed by annealing has the VBM at the binding energy of $1.7 \mathrm{eV}$. The obtained VBM value for the cleaned sample is in line with previous theoretical results. The energy distance between the Ga $3 d$ peak and the VBM is about $17.75 \mathrm{eV}$ for all the samples proving that the analyzed shifts of our XPS spectra result from electrostatic charge formed on the surface after certain treatments. The presence of oxygen and carbon contaminants on $p$-GaN(0001) surface changes the electrostatic charge of the sample surface, consequently leading to appearance of the inversion layer.

\section{Acknowledgments}

The authors would like to thank Aneta Mgłosiek for her help in the MBE laboratory. This work was supported by Foundation for Polish Science (FNP) project Team Tech/2016-3/16.

\section{References}

[1] Oxide and Nitride Semiconductors: Processing, Properties, and Applications, Eds. T. Yao, S.K. Hong, Springer, Berlin 2009.

[2] M.S. Shur, R. Gaska, A. Khan, Mater. Sci. Forum 353-356, 807 (2001).

[3] S. Nakamura, T. Mukai, M. Senoh, Appl. Phys. Lett. 64, 1687 (1994) 
[4] S. Nakamura, M. Senoh, S. Nagahama, N. Iwasa, T. Yamada, T. Matsushita, H. Kiyoku, Y. Sugimoto, Appl. Phys. Lett. 68, 2105 (1996).

[5] M. Asif Khan, Q. Chen, M.S. Shur, B.T. Dermott, J.A. Higgins, J. Burm, W.J. Schaff, L.F. Eastman, Solid-State Electron. 41, 1555 (1997).

[6] H. Matsubara, S. Yoshimoto, H. Saito, Y. Jianglin, Y. Tanaka, S. Noda, Science 319, 5862 (2008).

[7] S. Tripathy, S.J. Chua, A. Ramam, E.K. Sia, J.S. Pan, R. Lim, G. Yu, Z.X. Shen, J. Appl. Phys. 91, 3398 (2002).

[8] J. Sun, K.A. Rickert, J.M. Redwing, A.B. Ellis, F.J. Himpsel, T.F. Kuech, Appl. Phys. Lett. 76, 415 (2000).

[9] S.J. Pearton, J.C. Zolper, R.J. Shul, F. Ren, J. Appl. Phys. 86, 1 (1999).

[10] S.W. King, J.P. Barnak, M.D. Bremser, K.M. Tracy, C. Ronning, R.F. Davis, R. Nemanich, J. Appl. Phys. 84, 5248 (1998).

[11] B.S. Eller, J. Yang, R.J. Nemanich, J. Vac. Sci. Technol. A 31, 050807 (2013).

[12] J.J. Uhlrich, L.C. Grabow, M. Mavrikakis, T.F. Kuech, J. Electron. Mater. 37, 439 (2008).

[13] R.W. Hunt, L. Vanzetti, T. Castro, K.M. Chen, L. Sorba, P.I. Cohen, W. Gladfelter, J.M.V. Hove, J.N. Kuznia, M.A. Khan, A. Franciosi, Physica B Condens. Matter 185, 415 (1993).
[14] M. Grodzicki, P. Mazur, A. Ciszewski, Appl. Surf. Sci. 440, 547 (2018).

[15] H.W. Jang, J.L. Lee, J. Electrochem. Soc. 150, G513 (2003).

[16] I. Waki, H. Fujioka, K. Ono, M. Oshima, H. Miki, A. Fukizawa, Jpn. J. Appl. Phys. 39, 4451 (2000).

[17] J.R. Waldrop, R.W. Grant, Appl. Phys. Lett. 68, 2879 (1996)

[18] M. Grodzicki, P. Mazur, S. Zuber, J. Pers, A. Ciszewski, Mater. Sci. Pol. 32, 252 (2014).

[19] C.G.V. de Walle, D. Segev, J. Appl. Phys. 101, 081704 (2007).

[20] D. Segev, C.G.V. de Walle, EPL 76, 305 (2006).

[21] J.P. Long, V.M. Bermudez, Phys. Rev. B 66, 121308(R) (2002)

[22] Ł. Janicki, M. Gładysiewicz, J. Misiewicz, K. Klosek, M. Sobanska, P. Kempisty, Z.R. Zytkiewicz, R. Kudrawiec, Appl. Surf. Sci. 396, 1657 (2017).

[23] M. Grodzicki, P. Mazur, A. Ciszewski, Acta Phys. Pol. A 132, 351 (2017). 